\title{
Endosonographic detection of dumbbell-shaped jejunal GIST using double balloon enteroscopy
}

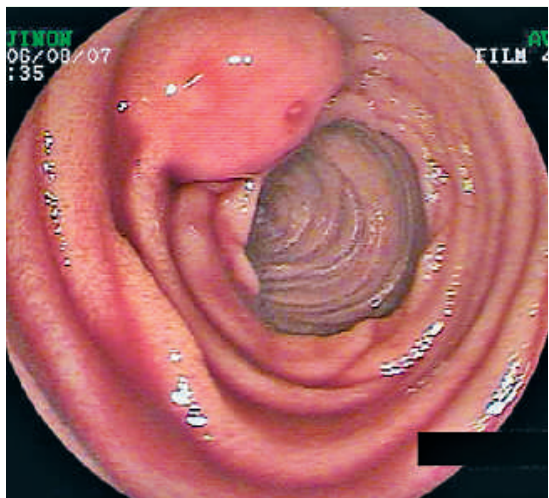

Fig. 1 Submucosal lesion was noted in the proximal jejunum.

The introduction of double balloon enteroscopy (DBE) into clinical practice has enabled us to examine lesions, even those located in the jejunum [1]. DBE has also been reported to be useful for the treatment of bleeding jejunal lesions [2]. We report a case of gastrointestinal stromal tumor (GIST) of the jejunum, a precise preoperative diagnosis of which was made with DBE.

A 48-year-old man was referred for evaluation of repeated bloody stools. The $\mathrm{Hb}$ level was $5.4 \mathrm{mg} / \mathrm{dL}$. Esophagogastroduodenoscopy, colonoscopy, and push enteroscopy (PCF P240 AI; Olympus, Tokyo, Japan) failed to reveal any obvious lesion. DBE (EN450 T5/20; Fujinon-Toshiba ES system, Tokyo, Japan) was carried out, and a submucosal lesion was noted in the jejunum, located approximately $1 \mathrm{~m}$ distal to the ligament of Treitz ( $\bullet$ Fig. 1). A small ulceration with active bleeding was noted at the top of the lesion. Hypertonic saline epinephrine injection was successfully carried out to terminate the bleeding. Endoscopic ultrasound with a 12-MHz US catheter probe (SP-702; Fujinon-Toshiba) disclosed a dumbbellshaped tumor in the jejunal wall (৫ Fig. 2). It measured $2 \mathrm{~cm}$ in diameter, with a homogeneous, hypoechoic pattern. It was contiguous with the proper

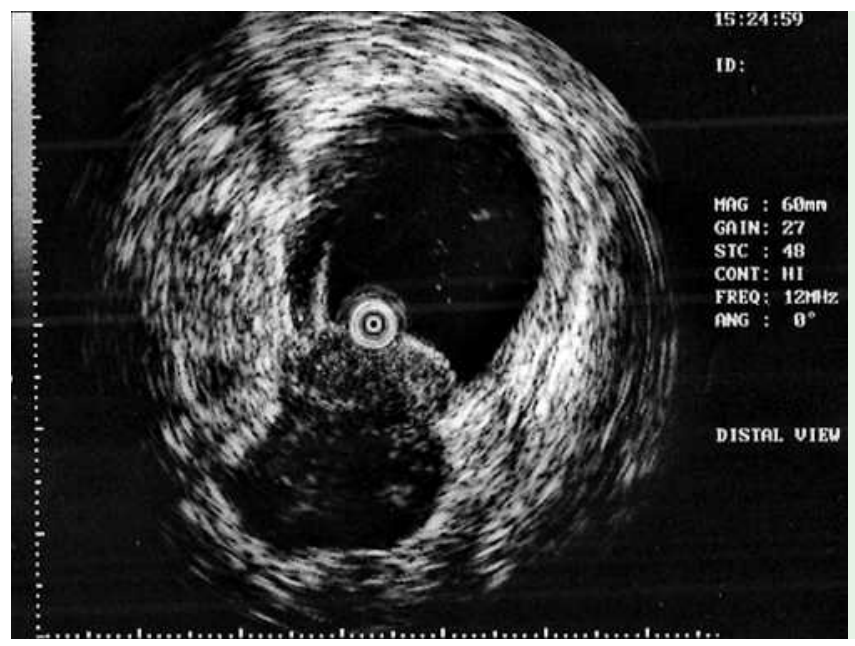

Fig. 2 Endoscopic ultrasound with a 12-MHz US catheter probe disclosed a dumbbell-shaped tumor in the jejunal wall.

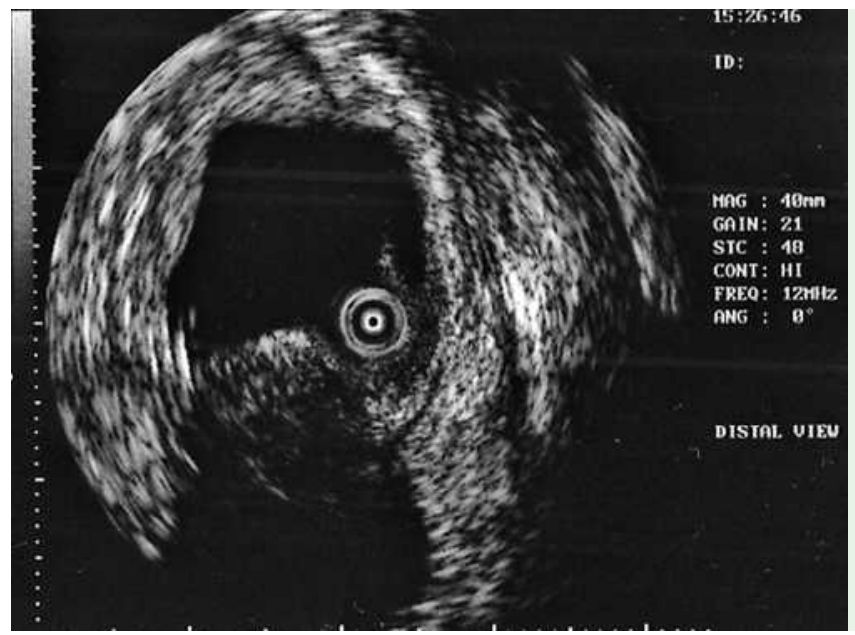

Fig. 3 The submucosal tumor was contiguous with the proper muscle layer.

muscle layer ( $\bullet$ Fig. 3). A preoperative diagnosis of jejunal GIST was made. The lesion was resected surgically.

The jejunal submucosal tumor was recognized from the outside of the lumen (- Fig. 4). The macroscopic view of the formalin-fixed specimen revealed clearly the dumbbell shape of the tumor (- Fig. 5); it measured $22 \times 19 \mathrm{~mm}$. Microscopically, the lesion was composed of interlacing bundles of spindle cells with no atypia, which derived from the proper muscle layer. No mitosis was noted (0/50 high-power field). Immunohis- tochemical study revealed positive staining of c-kit ( Fig. 6 ) and CD34. MIB-1 labeling index was $2 \%$. Postoperative histologic diagnosis was jejunal GIST, classified as low risk.

DBE is a useful modality for both diagnostic and therapeutic purposes.

Endoscopy_UCTN_Code_CCL_1AC_2AC 


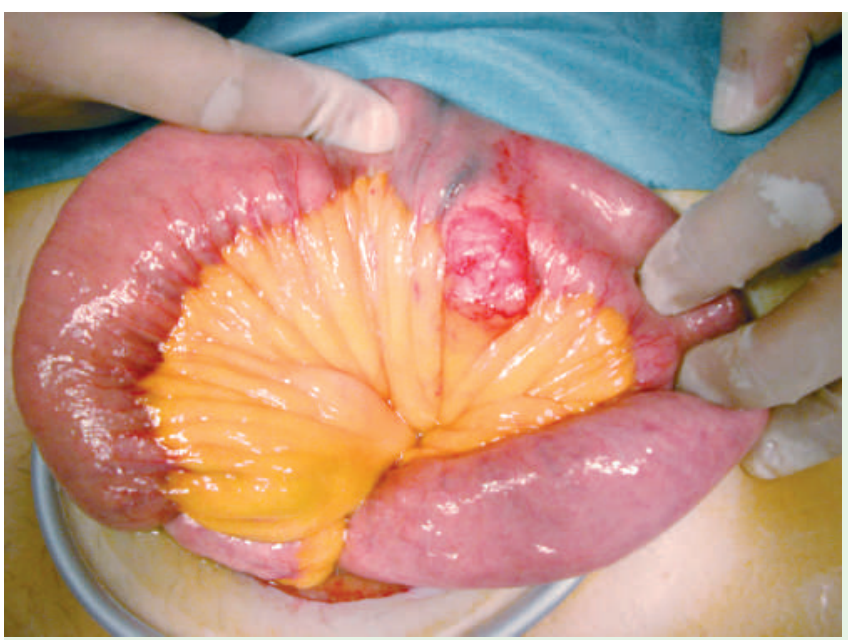

Fig. 4 Intraoperative view: the jejunal submucosal tumor was recognized from the outside of the lumen.

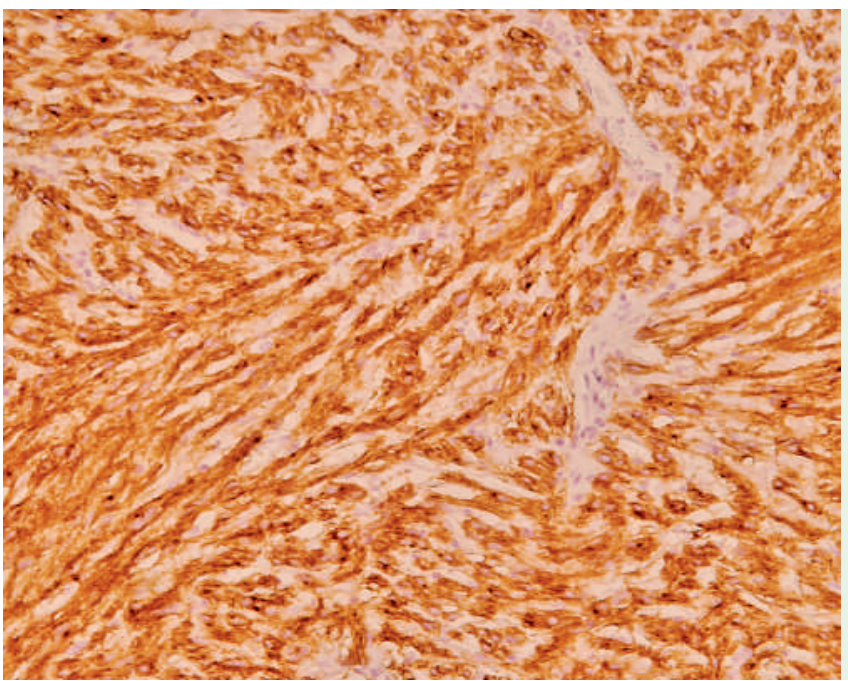

Fig. 6 Immunohistochemical study revealed positive staining of c-kit.

N. Matsui ${ }^{1}$, K. Akahoshi ${ }^{1}$, Y. Motomura ${ }^{1}$,

M. Kubokawa ${ }^{1}$, M. Kimura ${ }^{1}$, J. Ohuchi ${ }^{1}$,

K. Honda ${ }^{1}$, A. Murata ${ }^{1}$, S. Endoh' ${ }^{1}$,

M. Miyazaki², M. Oya ${ }^{3}$, S. Nakano ${ }^{4}$,

K. Nakamura ${ }^{5}$

1 Department of Gastroenterology, Aso lizuka Hospital, lizuka, Japan

2 Department of Surgery, Aso lizuka Hospital, lizuka, Japan

3 Department of Pathology, Aso lizuka Hospital, lizuka, Japan

4 Department of Gastroenterology, Shin Mizumaki Hospital, Fukuoka, Japan

${ }^{5}$ Department of Medicine and Bioregulatory Science, Graduate School of Medical Sciences, Kyushu University, Japan

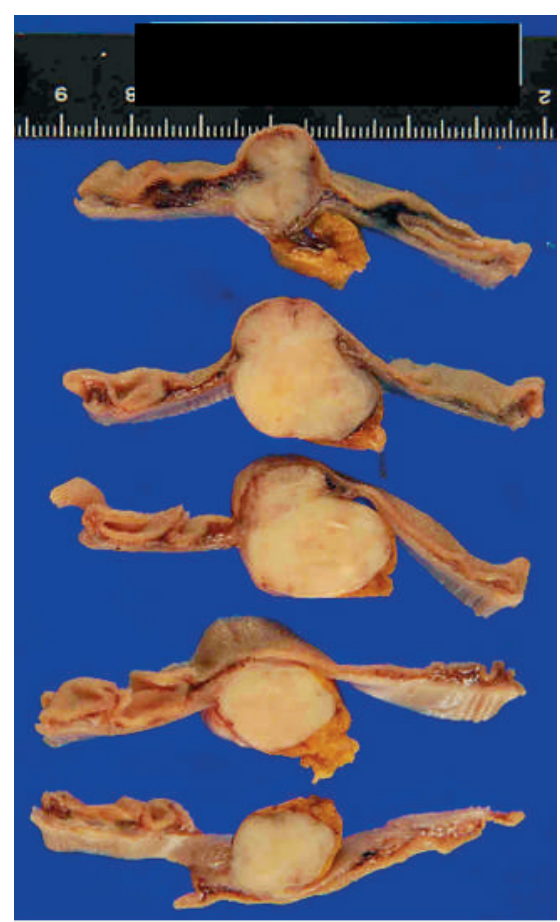

Fig. 5 Macroscopic view of the formalin-fixed specimen of the jejunal submucosal tumor.

Corresponding author

\section{N. Matsui, MD}

Department of Gastroenterology

Aso lizuka Hospital

3-83 Yoshio

lizuka 820-8505

Japan

Fax: + 81-948-29-8747

normatsui@yahoo.co.jp

\section{Bibliography}

Dol $10.1055 / \mathrm{s}-2007-966828$

Endoscopy 2008; 40: E38-E39

(c) Georg Thieme Verlag KG Stuttgart · New York . ISSN 0013-726X 